\title{
Copper-Rubber Interface Delamination in Stretchable Electronics
}

\author{
J. Neggers $^{1, \text { a }}$, J.P.M. Hoefnagels ${ }^{1}$, P.H.M. Timmermans ${ }^{2}$, and M.G.D. Geers ${ }^{1}$ \\ 1 Eindhoven University of Technology, Section Mechanics of Materials, P.O. Box 513, NL 5600 MB, \\ The Netherlands \\ 2 Philips Applied Technologies, System in Package,High Tech Campus 7, 5656 AE Eindhoven, The \\ Netherlands
}

\section{Introduction}

Interface delamination is a precursor to the failure of stretchable electronics mechanistic patterns of metal interconnects and elastically mismatched rubber matrix materials.

The goal of this project is to characterize interface delamination of the copper rubber interface by means of in-situ Environmental SEM analysis of the delamination front during $90^{\circ}$ Peel tests.

\section{Results}

From an analysis of systems of the same rubber but different $\mathrm{Cu}$ roughnesses, it was found that interfacial integrity is not only governed by chemical bonding, but also by;

- Local interface area (due to roughness)

- Mode mixity of the loading

- Mechanical interlocking

From a ESEM analysis of the delaminated surface of the same $\mathrm{Cu}$ (roughness) but 2 types of TPU (TU-Berlin) and 1 PDMS (IMEC Ghent) rubber system it was found that: The amount of rubber left at the surface $A_{r}$ after delamination remarkably increased with decreasing Work of Seperation (Figure 1).

From in-situ observations of the realtime delamination tests it was found that: Interface delamination is a delicate balance between the forming, elongation and rupture of fibrils and interface delamination (Figure 2).

\footnotetext{
a e-mail: j.neggers@tue.nl
} 
14th International Conference on Experimental Mechanics
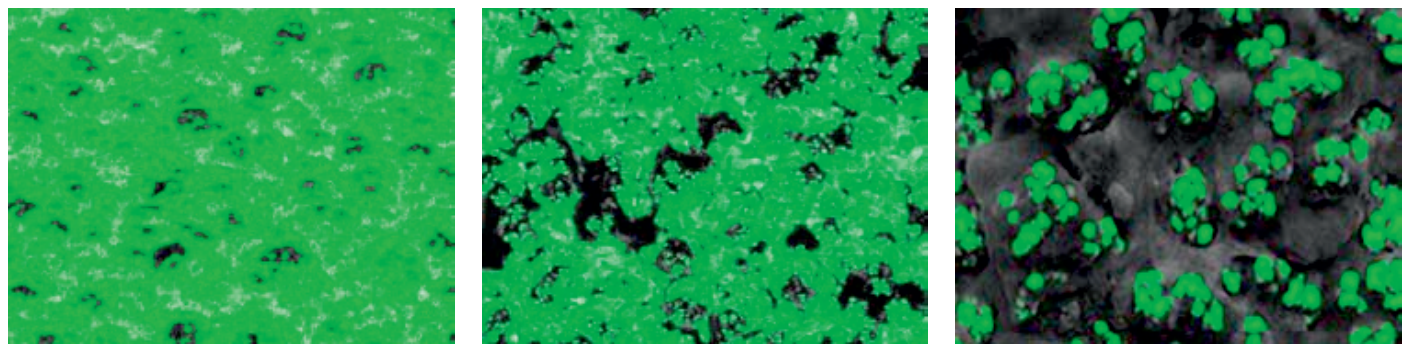
(a) TPU $180^{\circ}$,
$A_{r}=5.9 \%$,
$G_{c}=3.7 \mathrm{~kJ}$

(b) TPU $200^{\circ}$,

(c) PDMS,

$A_{r}=12 \%$,

$A_{r}=87 \%$,

$G_{c}=2.9 \mathrm{~kJ}$

$G_{c}=1.3 \mathrm{~kJ}$

Fig. 1: Analysis of ESEM images of the copper surface after peeling, rubber is shown in black and copper in green. With $A_{r}$ the rubber fraction and $G_{c}$ the Work of Seperation.

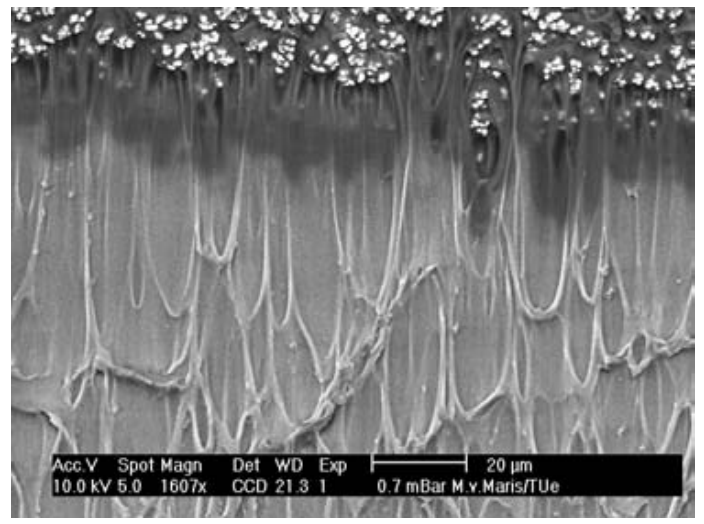

(a) PDMS

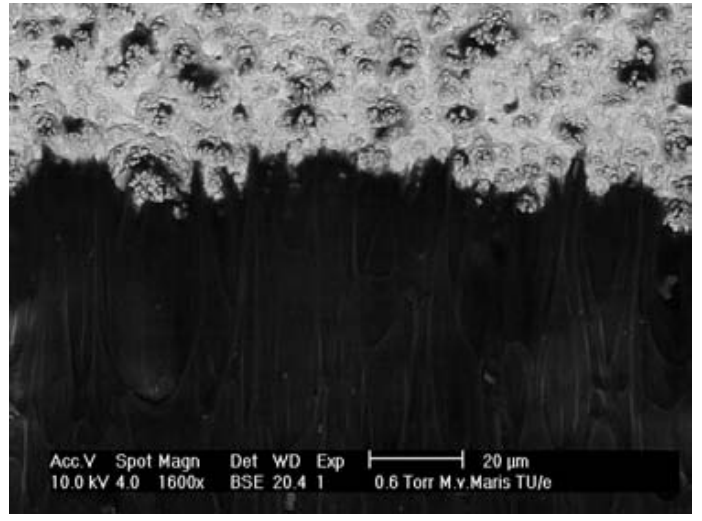

(b) TPU

Fig. 2: In-situ ESEM image of a progressing delamination front of the copper rubber interface.

T. Löher of TU-Berlin and J. Vanfleteren of IMEC Ghent are gratefully acknowledged for their work regarding sample preparation. 